\title{
Efecto potencial de la simvastatina en la regeneración tisular
}

\author{
Potential effect of simvastatin in tissue regeneration
}

\author{
ARDILA MEDINA CM*
}

Ardila Medina CM. Efecto potencial de la simvastatina en la regeneración tisular. Av Periodon Implantol. 2013; 25, 1: 11-16.

\begin{abstract}
RESUMEN
La simvastatina (SIM) es una lactona que se utilizan para disminuir el colesterol. Estudios in vitro y en animales han demostrado que induce la actividad osteoblástica y conduce a la formación ósea incrementando la expresión de la proteína morfogenética ósea-2. La SIM además de promover la formación ósea, suprime la síntesis de mevalonatos y pirofosfatos que a su vez inhiben la formación y actividad de los osteoclastos. La SIM es un potencial agente terapéutico para los pacientes con osteoporosis y puede facilitar el desarrollo de estrategias terapéuticas locales diseñadas para reparar anormalidades óseas como lesiones periodontales, lesiones periapicales, defectos del reborde alveolar y defectos óseos asociados a implantes. También se ha indicado como ingrediente activo para acelerar la diferenciación celular en la pulpa dental humana. Sin embargo, se requieren más ensayos clínicos controlados que corroboren los resultados obtenidos por las investigaciones realizadas en modelos animales de experimentación.
\end{abstract}

PALABRAS CLAVE: Simvastatina, regeneración tisular, diferenciación celular.

\section{SUMMARY}

Simvastatin (SIM) is a lactone which is used to lower cholesterol. Studies in vitro and in animals have been shown to induce osteoblastic activity and leads to bone formation by increasing the expression of bone morphogenetic protein-2. The SIM also promote bone formation, suppresses the synthesis of mevalonate and pyrophosphates, which in turn inhibit the formation and activity of osteoclasts. The SIM is a potential therapeutic agent for patients with osteoporosis and may facilitate the development of therapeutic strategies designed to repair local bone abnormalities such as periodontal lesions, periapical lesions, alveolar ridge defects and bone defects associated with implants. It was also indicated as the active ingredient to accelerate cell differentiation in human dental pulp. However, controlled clinical trials are required further corroborating the results of investigations in experimental animal models.

KEY WORDS: Simvastatin, tissue regeneration, cell differentiation.

Fecha de recepción: 10 de diciembre de 2009.

Fecha de aceptación: 25 de enero de 2010.

\section{INTRODUCCIÓN}

Las estatinas son inhibidores competitivos de la 3hidroxi-3-metilglutaril coenzima A reductasa (HMGCoA). Estas incluyen la lovastatina, sus modificaciones químicas (simvastatina y pravastatina) y derivados sintéticos (atorvastatina, fluvastatina y cerivastatina) ( 1 , 2). Estos agentes se utilizan para disminuir el colesterol, proporcionando un enfoque efectivo para el tratamiento de la hiperlipidemia y la arteriosclerosis $(1,2)$. 
Adicionalmente, estudios in vitro y en animales han demostrado que inducen la actividad osteoblástica y conducen a la formación ósea incrementando la expresión de la proteína morfogenética ósea-2 (PMO-2) $(3,4)$. También promueven la vasculogénesis y tienen efectos antiinflamatorios e inmunomoduladores $(3,4)$.

La simvastatina (SIM) es una lactona inactiva que después de administrarse oralmente se convierte en su forma activa por acción de enzimas intracelulares (5). Desafortunadamente, menos del 5\% de las dosis orales de SIM alcanzan la circulación sistémica (6), sin embargo, las células óseas se pueden exponer a muy bajas concentraciones de estatinas con las dosificaciones existentes (6). La SIM además de promover la formación ósea $(7,8)$, suprime la síntesis de mevalonatos y pirofosfatos que a su vez inhiben la formación y actividad de los osteoclastos (9). De esta manera, las estatinas pueden incrementar la masa ósea mediante mecanismos anabólicos y anticatabólicos (10). Además, la administración de estatinas combinadas con terapias para la osteoporosis parece mejorar la arquitectura ósea alveolar en maxilares de animales experimentales (11). De esta manera la SIM es un potencial agente terapéutico para los pacientes con osteoporosis y puede facilitar el desarrollo de estrategias terapéuticas locales diseñadas para reparar anormalidades óseas como lesiones periodontales, lesiones periapicales, defectos del reborde alveolar y defectos óseos asociados a implantes (11-14). Recientemente, también se ha indicado como ingrediente activo para acelerar la diferenciación celular en la pulpa dental humana (15).

El objetivo de este artículo es presentar el uso potencial de la simvastatina en la regeneración tisular.

\section{ESTUDIOS IN VITRO}

Se han realizado diferentes estudios utilizando células humanas $(16,17)$ con el fin de evaluar los efectos antiinflamatorios, proliferación celular y diferenciación osteoblástica producidos por la SIM. Es así como Sakoda y cols. (16) estudiaron el efecto antiinflamatorio de la SIM en los tejidos orales utilizando células epiteliales gingivales KB. La citotoxicidad de la SIM se evaluó mediante una enzima citoplasmática. Se midieron también los efectos de la SIM sobre la producción de interleuquina- 6 y 8 . Esta investigación fue la primera en demostrar que la SIM reduce la producción de citoquinas inflamatorias involucrando mecanismos independientes al de la disminución del co- lesterol. Estudios similares han demostrado también los efectos pleiotrópicos de la SIM sobre otro tipo de células como las endoteliales, miocitos cardíacos y macrófagos (18).

Otro estudio in vitro como el realizado por Yazawa y cols. (17) ha confirmado el efecto positivo de la SIM sobre la formación ósea. Estos investigadores fueron los primeros en evaluar el efecto de la SIM sobre las células del ligamento periodontal enfocándose específicamente en la proliferación celular, metabolismo y potencial de mineralización. Es importante tener en cuenta que las células del ligamento periodontal estudiadas se tomaron de pacientes con tejidos periodontales saludables. Después de 24 horas de incubación y antes de 72 horas, la SIM aumentó la proliferación celular, el metabolismo y diferenciación de los osteoblastos, estimulando también la actividad de la fosfatasa alcalina, la osteopontina y el contenido de calcio. Sin embargo, los investigadores no detectaron contenido de PMO-2 ni de osteocalcina, y en concentraciones de SIM mayores a $10^{-6}$, se inhibió la proliferación celular y el metabolismo de las células del ligamento periodontal a las 72 horas. En estudios previos al realizado por Yazawa y cols. (17), el efecto de la SIM sobre la proliferación y metabolismo celular también mostró variaciones de acuerdo al tipo de célula, tipo de estatina y concentración $(19,20)$. La tabla 1 presenta los efectos terapéuticos de la SIM en algunos estudios in vitro.

\section{ESTUDIOS IN VIVO}

En odontología se han utilizado modelos animales experimentales con el fin de evaluar los efectos de la SIM (10, 13-15, 21). Estos estudios han investigado sus propiedades regenerativas óseas en defectos óseos periodontales, periapicales y alrededor de implantes (13, 15, 21-27). Además, se ha estudiado su capacidad para mantener rebordes alveolares después de exodoncias y su participación en la diferenciación celular en la pulpa dental humana (15). También se ha evaluado la SIM en combinación con otros agentes y se han valorado sus resultados en distracción osteogénica (28-30). Solamente un estudio clínico, realizado en pacientes con periodontitis crónica, ha examinado su potencial regenerativo en defectos óseos periodontales (12). La tabla l presenta los efectos potenciales de la SIM observados en algunos estudios in vivo.

Bradley y cols. (21) realizaron un estudio con el fin de confirmar el conocimiento aportado por estudios in 


\section{TABLA 1.- EFECTOS DE LA SIMVASTATINA COMO REGENERADOR TISULAR}

\begin{tabular}{|l|l|c|}
\hline Efecto & Tipo de estudio & Referencia \\
\hline Antiinflamatorio & In vitro & $(16)$ \\
\hline Proliferación celular & In vitro & $(17)$ \\
\hline Diferenciación de osteoblastos & In vitro & $(21)$ \\
\hline Formación ósea & In vivo en ratas & $(22)$ \\
\hline Nuevo cemento & In vivo en perros & $(22)$ \\
\hline Aumento de Reborde & In vivo en ratas & $(23),(26)$ \\
\hline Osteogénesis sobre implantes & In vivo en perros y ratas & $(27)$ \\
\hline Preservación del reborde alveolar & In vivo en ratas & $(28)$ \\
\hline $\begin{array}{l}\text { Incremento de la regeneración ósea en distracción } \\
\text { osteogénica }\end{array}$ & In vivo en conejos & $(15)$ \\
\hline Diferenciación de células pulpares & In vivo en molares humanos & $(13)$ \\
\hline Disminución de lesión periapical & In vivo en ratas & \\
\hline $\begin{array}{l}\text { Reducción del sangrado gingival, profundidad al sondaje } \\
\text { y defectos óseos. } \\
\text { Ganancia en el nivel de inserción }\end{array}$ & & Ensayo clínico controlado \\
\hline
\end{tabular}

vitro $(3,4)$ relacionado con el incremento de la expresión de la PMO-2 durante la formación ósea inducida por la SIM. Se utilizó un modelo bilateral en ratas aplicando $0,5 \mathrm{mg}$ de SIM. Sus resultados confirmaron la capacidad de la SIM para inducir PMO-2 y formación ósea en microambientes mandibulares.

Morris y cols. (22) crearon defectos periodontales bilaterales en perros beagle con el fin de evaluar el efecto de la SIM inyectada localmente. Después de 16 semanas de cicatrización, los sitios fueron tratados con terapia mecánica periodontal y las mandíbulas se asignaron aleatoriamente para recibir tres inyecciones semanales de 0,5 mg de SIM en $30 \mu \mathrm{l}$ de metilcelulosa en gel o solo gel en lado contralateral. Dos meses después de la aplicación de los agentes, se realizaron secciones en bloque incluyendo dientes y tejidos. El espesor del reborde edéntulo bucal tratado con SIM de $0,5 \mathrm{mg}$ aumento $29 \%$. La longitud del nuevo cemento en los defectos óseos interproximales también fue superior en el grupo tratado con SIM de $0,5 \mathrm{mg}(0,35 \pm$ $0,14 \mathrm{~mm}$ ). Sin embargo no se encontró nuevo cemento en las furcaciones.
También se ha investigado la capacidad de la SIM para formar hueso alrededor de implantes de titanio (2325). Recientemente, Ayukawa y cols. (23) estudiaron 30 ratas que recibieron implantes en sus dos tibias. Se les aplicó diaria e intraperitonealmente 0, 0,125, 1, 5 ○ $10 \mathrm{mg} \mathrm{kg}^{-1}$ de SIM. Se observó mayor espesor de hueso trabecular alrededor del titanio en los grupos tratados con 5 y $10 \mathrm{mg} \mathrm{kg}^{-1}$, pero no se encontraron diferencias en el porcentaje de hueso cortical entre los grupos. Estos resultados corroboraron los obtenidos por estudios previos $(24,25)$ en donde la administración de $10 \mathrm{mg} \mathrm{kg}^{-1}$ de SIM promovió la osteogénesis incrementando también el volumen del hueso trabecular. Duz y cols. (26) estudiaron el efecto de la SIM sobre la cicatrización ósea alrededor de implantes colocados en ratas con osteoporosis. Estos investigadores encontraron que la SIM incrementa la taza de contacto hueso implante y el volumen de hueso cancelar, permitiendo estabilidad secundaria. Sus resultados sugieren que la SIM puede sobreponer efectivamente el impacto negativo que ejerce la osteoporosis sobre la oseointegración de implantes de titanio. 
Por otra parte, Wu y cols. (27) evaluaron el efecto de la SIM sobre la reabsorción del reborde residual posterior a exodoncias. Los investigadores implantaron medios de transporte polilácticos con y sin SIM en alveolos mandibulares de ratas Wistar. La altura del reborde alveolar residual y la densidad mineral del hueso fueron mayores en el grupo experimental comparado con el grupo control, concluyendo de esta manera que la SIM podría preservar el reborde alveolar residual después de las extracciones dentales.

Kiliç y cols. (28) estudiaron los efectos de la aplicación local y sistémica de la SIM sobre la distracción osteogénica. Se aplicó distracción a 18 conejos neozelandeses durante 10 días. La SIM se aplicó localmente por medio de una esponja durante la fase de osteotomía, y sistémicamente mediante sonda durante el período de distracción. La evaluación radiográfica computarizada reveló un incremento del 9,6 \% y 19,3\% en la regeneración, después de aplicarse SIM en forma local y sistémica respectivamente.

En el área de la endodoncia la SIM ha mostrado resultados importantes. Okamoto y cols. (15) estudiaron el efecto de la SIM sobre la diferenciación de las células pulpares en siete molares de cinco adultos. La reacción en cadena de la polimerasa (PCR) reveló que cuando las células madres de la pulpa dental humana se cultivaron conjuntamente con SIM, la osteocalcina y la dentina se regularon de forma significativa. Los investigadores también encontraron que la SIM promueve una formación tisular mineralizada, sugiriendo que esta estatina puede ser un ingrediente activo para acelerar la diferenciación de las células pulpares, indicando de esta forma un posible efecto regenerativo en la pulpa y dentina. Además, el efecto antiinflamatorio de la SIM ayudaría a restaurar los tejidos inflamados de la pulpa. Por su parte, Lin y cols. (13) evaluaron el efecto terapéutico de la SIM en periodontitis apical inducida en 20 ratas Sprague. Las evaluaciones radiográficas e histológicas mostraron que la administración de SIM disminuía notablemente la severidad de las lesiones periapicales posiblemente por la reducción de la expresión de la cisteína $\mathrm{Gl}$ en los osteoblastos y consecuentemente la quimiotaxis de los macrófagos en las lesiones.

Solamente un ensayo clínico controlado realizado en pacientes con periodontitis ha mostrado resultados relevantes de la SIM como regenerador óseo (12). Pradeep y cols. (12) evaluaron la eficacia clínica y radiográfica de la aplicación de 1,2 mg de SIM como terapia adjunta al raspado y alisado radicular en pacien- tes con periodontitis crónica. Un total de 60 pacientes fueron asignados aleatoriamente a dos tipos de terapia. El primer grupo recibió terapia mecánica y el segundo terapia mecánica más SIM. En los pacientes del grupo experimental se observó una mayor reducción en el sangrado del surco gingival, en la profundidad al sondaje y en los defectos intraóseos. Igualmente se encontró mayor ganancia de inserción clínica en el grupo intervenido con SIM. Los autores concluyeron que estas observaciones pueden proporcionar una nueva orientación en el área de la regeneración periodontal.

Finalmente, el desarrollo de materiales de injertos óseos con propiedades osteogénicas ha motivado la experimentación con diferentes agentes. Es así como la SIM en combinación con sulfato de calcio y fosfato tricálcico han mostrado estimulación de la regeneración ósea y podrían ser aplicados como material de injerto óseo $(29,30)$.

\section{CONCLUSIONES}

Diferentes estudios preclínicos y clínicos indican el posible beneficio de la simvastatina como regenerador tisular. Los estudios in vitro e in vivo demuestran que la SIM promueve la diferenciación de osteoblastos como es evidenciado por la expresión de PMO-2 y el aumento de la mineralización. Además, parece que acelera la diferenciación de las células madre de la pulpa dental. Se requieren más ensayos clínicos controlados que corroboren los resultados obtenidos por las investigaciones realizadas en modelos animales de experimentación.

\section{BIBLIOGRAFÍA}

1. Henwood JM, Heel RC. Lovastatin. A preliminary review of its pharmacodynamic properties and therapeutic use in hyperlipidaemia. Drugs 1988; 36:429-54.

2. Todd PA, Goa Kl. Simvastatin. A review of its pharmacological properties and therapeutic potential in hypercholesterolaemia. Drugs 1990; 40:583-607.

3. Mundy G, Garrett R, Harris S, Chan J, Chen D, Rossini G, et al. Stimulation of bone formation in vitro and in rodents by statins. Science 1999; 286:1946-9.

4. Whang K, Zhao M, Qaio M, Rossini G, Horn D, Garrett IR, et al. Administration of lovastatin locally in low doses 
in a novel; delivery system induces prolonged bone formation. J Bone Miner Res 2000; 15(Suppl):S225.

5. Jadhav SB, Jain GK. Statins and osteoporosis: new role for old drugs. J Pharm Pharmacol 2006;58:3-18.

6. Park JB. The use of simvastatin in bone regeneration. Med Oral Patol Oral Cir Bucal 2009;14:e485-8.

7. Garrett IR, Gutierrez G, Mundy GR. Statins and bone formation. Curr Pharm Des 2001;7:715-36.

8. Maeda T, Matsunuma A, Kurahashi I, Yanagawa T, Yoshida $\mathrm{H}$, Horiuchi $\mathrm{N}$. Induction of osteoblast differentiation indices by statins in MC3T3-El cells. J Cell Biochem 2004;92:458-71.

9. Hughes A, Rogers MJ, Idris AI, Crockett JC. A comparison between the effects of hydrophobic and hydrophilic statins on osteoclast function in vitro and ovariectomyinduced bone loss in vivo. Calcif Tissue Int 2007;81:40313.

10. Jeon JH, Piepgrass WT, Lin YL, Thomas MV, Puleo DA. Localized intermittent delivery of simvastatin hydroxyacid stimulates bone formation in rats. J Periodontol 2008;79:1457-64.

11. Horiuchi N, Maeda T. Statins and bone metabolism. Oral Dis 2006;12:85-101.

12. Pradeep AR, Manojkumar T. Clinical Effect of Subgingivally Delivered Simvastatin in the Treatment of Chronic Periodontitis Patients: A Randomized Clinical Trial. J Periodontol 2009 (in press). DOI: 10.1902/ jop.2009.090429.

13. Lin SK, Kok SH, Lee YL, Hou KL, Lin YT, Chen MH, et al. Simvastatin as a novel strategy to alleviate periapical lesions. J Endod 2009;35:657-62.

14. Nishimura K. Local application of simvastatin to rat incisor sockets augments bone. Kokubyo Gakkai Zasshi 2008;75:49-54.

15. Okamoto Y, Sonoyama W, Ono M, Akiyama K, Fujisawa T, Oshima M, et al. Simvastatin induces the odontogenic differentiation of human dental pulp stem cells in vitro and in vivo. J Endod 2009;35:367-72.

16. Sakoda K, Yamamoto M, NegishiY, Liao JK, Node K, Izumi Y. Simvastatin decreases IL-6 and IL-8 production in epithelial cells. J Dent Res 2006; 85:520-3.
17. Yazawa H, Zimmermann B, Asami Y, Bernimoulin JP. Simvastatin promotes cell metabolism, proliferation and osteoblastic differentiation in human periodontal ligament cells. J Periodontol 2005;76:295-302.

18. Takemoto M, Liao JK. Pleiotropic effects of 3-hydroxy3-methylglutaryl coenzyme a reductase inhibitors. Arterioscler Thromb Vasc Biol 2001;21:1712-19.

19. Axel DI, Riessen R, Runge H, Viebahn H, Karsch KR. Effects of cerivastatin on human arterial smooth muscle cell proliferation and migration in transfilter cocultures. J Cardiovasc Pharmacol 2000;35:619-29.

20. Vincent L, Chen W, Hong L. Inhibition of endothelial cell migration by cerivastatin , an HMG-CoA reductase inhibitors. FEBS Lett 2001;495:159-66.

21. Bradley JD, Cleverly DG, Burns AM, Helm NB, Schmid MJ, Marx DB, et al. Cyclooxygenase-2 inhibitor reduces simvastatin-induced bone morphogenetic protein-2 and bone formation in vivo. J Periodontal Res 2007;42:26773.

22. Morris MS, Lee Y, Lavin MT, Giannini PJ, Schmid MJ, Marx $\mathrm{DB}$, et al. Injectable simvastatin in periodontal defects and alveolar ridges: pilot studies. J Periodontol 2008;79:1465-73.

23. Ayukawa Y, Ogino Y, Moriyama Y, Atsuta I, Jinno Y, Kihara $\mathrm{M}$, et al. Simvastatin enhances bone formation around titanium implants in rat tibiae. J Oral Rehabil 2009 (in press).

24. Oxlund H, Dalstra M, Andreassen TT. Statin given perorally to adult rats increases cancellous bone mass and compressive strength. Calcif Tissue Int 2001;69:299304.

25. Ayukawa Y, Okamura A, Koyano K. Simvastatin promotes osteogenesis around titanium implants. Clin Oral Implants Res 2004;15:346-50.

26. Du Z, Chen J, Yan F, XiaoY. Effects of Simvastatin on bone healing around titanium implants in osteoporotic rats. Clin Oral Implants Res 2009;20:145-50.

27. Wu Z, Liu C, Zang G. The effect of simvastatin on remodelling of the alveolar bone following tooth extraction. Int J Oral Maxillofac Surg 2008; 37:170-6.

28. Kiliç E, Ozeç I, Yeler H, Korkmaz A, Ayas B, Gümüş C. Effects of simvastatin on mandibular distraction 


\section{AVANCES}

Volumen 25 - No 1 - Abril 2013

osteogenesis. J Oral Maxillofac Surg 2008;66:22338.

29. Nyan M, Sato D, Oda M, Machida T, Kobayashi H, Nakamura T, et al. Bone formation with the combination of simvastatin and calcium sulfate in critical-sized rat calvarial defect. J Pharmacol Sci 2007;104:384-6.

30. Nyan M, Sato D, Kihara H, Machida T, Ohya K, Kasugai S. Effects of the combination with alpha-tricalcium phosphate and simvastatin on bone regeneration. Clin Oral Implants Res 2009;20:280-7.

\section{CORRESPONDENCIA}

Carlos Martín Ardila Medina

Calle 64, n 52-59

Medellín, Antioquía, Colombia.

Correo electrónico: martinardila@gmail.com 